\title{
HIGHLIGHTS
}

\section{Patient-controlled sedation shows promise for colonoscopy}

Patient-controlled sedation (PCS) with propofol and remfentanil is less likely to be associated with intervention for hypoventilation than anesthesiologistadministered sedation (AAS) with these agents, according to a new study by Jeff Mandel and colleagues.

The use of propofol plus remfentanil for sedation of patients undergoing colonoscopy is increasing, but the administration of these agents is restricted to anesthesiologists. This requirement has raised concerns about the cost of these procedures."With 30 million colonoscopies performed in the US annually, the economic impact is staggering," says Mandel, lead author of the study.

Their prospective, randomized, open-label study included 50 patients undergoing elective colonoscopy. All patients were moderately sedated before receiving PCS or AAS for the duration of the procedure. For PCS, the patient was able to control sedation on demand-in doses of $3 \mathrm{mg}$ of propofol plus $3 \mu \mathrm{g}$ remfentanil with at least a $6 \mathrm{~s}$ fixed interval period between doses. AAS involved the use of software designed to assist the anesthesiologist in providing moderate sedation by predicting drug response in the patient over the next $90 \mathrm{~s}$.

"If you consider this to be a game..." explains Mandel "...where the objective is to minimize hypoventilation while not having the patient undersedated, the patient has the advantage of more accurate information on the effect of the drug on their sensorium, and the anesthesiologist has the advantage of more accurate information on the future time course of drug levels."

Oversedation was far more common in the AAS group than the PCS group. Five patients in the AAS group required airway rescue compared with none in the PCS group. The rate of respiratory events was significantly lower when the patient, rather than the anesthetist, controlled the administration of sedation.

"The advantage of having the patient in the loop is hard to overcome," says Mandel. He is keen to explain the potential economic benefits of such a strategy. "PCS could be employed in a reduced staffing model, for example, where one

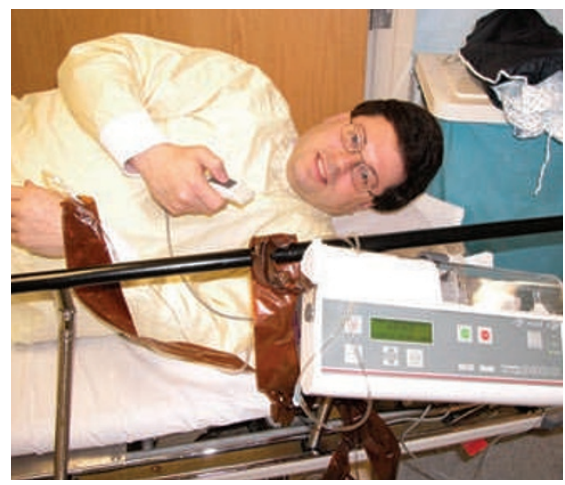

Author Gary Lichtenstein simulating use of patient-controlled sedation.

anesthesiologist supervises more than one patient in a multiroom suite. This model would permit the use of propofol in a more cost-effective fashion, and avoid the existing fight over the labeling of propofol for use by practitioners trained in rescue from unintended general anesthesia."

Mandel is now in the process of designing new software to improve PCS by incorporating predictive drug response systems with patient control.

Rachel Jones

Original article Mandel, J. E. et al. A prospective,
randomized, comparative trial evaluating respiratory
depression during patient-controlled versus
anaesthesiologist-administered propofol-remifentanil
sedation for elective colonoscopy. Gastrointest. Endosc. 72,
112-117 (2010)

